\title{
Mechanisms of second and further strokes
}

\author{
Haruko Yamamoto, Julien Bogousslavsky
}

\begin{abstract}
Objectives-The mechanisms underlying recurrent stroke may be complex and multifactorial, but they have not been studied systematically. The aim was to analyse the different patterns and pathophysiological mechanisms of second and further strokes.
\end{abstract}

Methods-Recurrent stroke patterns and mechanisms were studied in 102 patients admitted with second or further strokes to the stroke centre in Lausanne University Hospital.

Results-The patients with an initial cardioembolic stroke experienced recurrent stroke of the same type most often, followed by those with initial non-lacunar non-cardioembolic stroke, brain haemorrhage, and lacunar stroke $(77 \%, 65 \%, 58 \%$, and $48 \%$ respectively). Forty two per cent of the recurrent strokes in patients with an initial brain haemorrhage were ischaemic, whereas patients with ischaemic stroke only occasionally suffered brain haemorrhage $(5 \%)$. In patients with brain haemorrhage, the lobar location predominated in both the first and all episodes (69\% and $78 \%$ respectively), suggesting a small, occult arteriovenous malformation or cerebral amyloid angiopathy rather than hypertensive small artery disease. The functional disability of patients after an initial lacunar stroke was significantly better than in patients with other stroke subtypes $(p<0.001)$, but the difference became non-significant after recurrent stroke $(\mathrm{p}=0.26)$.

Conclusions-Most of the recurrent strokes were of the same type as the first episode for both cardioembolic and nonlacunar non-cardioembolic stroke, however, about half of the patients with an initial brain haemorrhage or a lacunar stroke experienced other types of stroke recurrently. The findings suggest that the coexistence of multiple aetiologies may play a major part in determining the mechanism of stroke recurrence. The study is an important step in understanding the patterns of stroke recurrence, which may be critical for better prevention. (f Neurol Neurosurg Psychiatry 1998;64:771-776)

Keywords: cerebral infarction; intracerebral haemorrhage; recurrence

Correspondence to: Professor Julien B

Bogousslavsky, Service de Neurologie, Centre Hospitalier Universitaire Vaudois-BH13, CH-1011

Lausanne, Switzerland.

Received 17 July 1997 and in final revised form 9 March 1998

Accepted 11 March 1998 ment of these risk factors can prevent stroke recurrence, ${ }^{212}$ probably because the mechanisms underlying recurrent stroke are complex and multifactorial. Moreover, a precise aetiological classification of recurrent stroke may be difficult in such epidemiological studies with many patients (cardioembolic infarction after lacunar stroke or brain haemorrhage after large artery disease, etc). For example, in the Stroke Data Bank, ${ }^{6}{ }^{7}$ ischaemic strokes are subdivided into six aetiologies: lacunar stroke, cardiac embolism, large artery atherosclerosis, tandem pathology, other unusual causes, and undetermined cause. Although a predominance of same type recurrence after large artery atherosclerosis has been reported, about half of the patients with stroke recurrence of the same type are in fact classified into the group with strokes of undetermined cause. Understanding the pathophysiological mechanisms of stroke recurrence is mandatory for effective secondary prevention. The aim of this study was to analyse the different aetiological patterns and underlying mechanism of recurrence after an initial stroke.

\section{Methods}

From January 1990 to November 1995 we studied patients admitted to the stroke centre at Lausanne University Hospital with recurrent stroke (acute brain infarction or intracerebral haemorrhage) after a well identified initial stroke. Lausanne University Hospital is the only public hospital in the Lausanne area and is located in a city of 250000 people. Most acute patients in this area are referred to the stroke centre at Lausanne University Hospital. We excluded patients: (1) without detailed medical records or without CT or MRI in the acute stage of the first stroke, so that the type of stroke could be differentiated; (2) with stroke after medical procedures (carotid endarterectomy, angiography, etc) or trauma; (3) with cerebral haemorrhage which was intratumorous or caused by a ruptured cerebral aneurysm, or vascular malformation; and (4) with subarachnoid haemorrhage or subdural haemorrhage. We also excluded patients with a recurrence of only transient ischaemic attacks (TIAs) because it was difficult to determine the aetiology of the TIA episodes from limited information. Information about the first strokes of patients who had not been admitted in our service was collected from their families, home doctors, and hospitals where they were admitted during their first episodes. Systematic investigations were performed following the standard protocol of the Lausanne Stroke Registry, ${ }^{13}$ including brain CT, carotid ultrasound, transcranial Doppler, 12 lead ECG, three lead ECG monitoring (24 to 70 hours), and standard blood and urine tests, including 
Table 1 General status of the patients

\begin{tabular}{llllll}
\hline 1st stroke & NL-CE & NL-NE & $L I$ & BH & Total \\
\hline Total (n) & 20 & 47 & 19 & 16 & 102 \\
Age (mean (SD)) & $68.3(11.0)$ & $63.0(14.0)$ & $60.7(14.7)$ & $63.6(10.5)$ & $63.7(13.2)$ \\
Male (n) & 15 & 36 & 13 & 13 & 77 \\
Age (mean (SD)) & $67.7(11.6)$ & $64.1(10.3)$ & $60.8(11.4)$ & $62.7(10.8)$ & $64.0(10.8)$ \\
Female (n) & 5 & 11 & 6 & 3 & 25 \\
Age (mean (SD)) & $70.0(10.1)$ & $59.4(22.6)$ & $60.7(21.6)$ & $67.3(10.1)$ & $62.8(18.9)$ \\
Interval (mean (SD), months) & $31.4(29.5)$ & $38.5(30.3)$ & $68.5(56.7)$ & $38.6(49.5)$ & \\
\hline
\end{tabular}

NL-CE=non-lacunar cardioembolic infarction; NL-NE=non-lacunar non-cardioembolic infarction; LI=lacunar infarction; $\mathrm{BH}=$ brain haemorrhage; 1 st stroke=the type of the first stroke; mean interval=the number of months between the first and the last stroke episode.

venous packed cell volume and fasting blood cholesterol. Cerebral angiography, brain MRI, two dimensional transthoracic and transoesophageal echocardiography with or without a microbubble test, and 24 hour ECG (Holter) monitoring were performed in selected patients.

Strokes were classified into three main groups based on the definitions of the Lausanne Stroke Registry ${ }^{13}$ : (1) lacunar infarction (infarct with a lacunar syndrome (pure motor hemiparesis, pure sensory stroke, sensory-motor stroke, and ataxic hemiparesis) and a normal CT or small ( $\leqslant 15 \mathrm{~mm})$, deep infarct on CT); (2) non-lacunar infarction (infarct with neurological findings not compatible with lacunar syndrome and a normal CT or a hypodense area $>15 \mathrm{~mm}$, corresponding to the clinical picture); and (3) brain haemorrhage (stroke with a hyperdense area suggesting primary bleeding corresponding to the clinical picture, excluding haemorrhagic transformation of ischaemic stroke). The presumed causes of non-lacunar infarction included ${ }^{13}:$ (1) large artery atheroma with $>50 \%$ stenosis of the corresponding artery (extracranial or intracranial); (2) atherosclerosis, but without $>50 \%$ stenosis of the corresponding large artery; (3) cardiac embolic sources (mitral or aortic valvar diseases including mitral valve prolapse, prosthetic valves, recent myocardial infarction, infective or non-infective endocarditis, non-ischaemic dilated cardiomyopathy, left ventricular ischaemic dyskinesia, left ventricular thrombus, atrial myxoma, atrial fibrillation, sick sinus syndrome, and patent foramen ovale with or without atrial septal aneurysm, with presumed paradoxical embolism); and (4) other causes, which were not compatible with large artery atheroma or cardiac embolic sources, or which remained undetermined. In the present study, non-lacunar infarctions were subdivided according to the presence or absence of cardiac embolic sources. However, infarcts occurred in the internal carotid artery area of the occluded side, and infarcts in the border zone suspected of a haemodynamic mechanism because of an associated decrease in blood pressure were classified as noncardioembolic infarcts. Infarct topography was classified as: (1) anterior circulation (including superficial and deep territories); (2) posterior circulation; and (3) border zone according to reported guidelines. ${ }^{14}$ Brain haemorrhage was subdivided into two aetiological subtypes: (1) hypertensive and (2) non-hypertensive according to history of hypertension. We considered two topographic forms of haemorrhage: (1) lobar (supratentorial) and (2) non-lobar, including the thalamus, basal ganglia, and infratentorial. The classification was performed independently by the two authors in a non-blind fashion to the subtypes of the patients' other stroke episodes. If different classifications were made, we discussed these differences until accordance was achieved.

The following risk factors were considered ${ }^{13}$ : (1) hypertension (blood pressure $>160 / 90 \mathrm{~mm}$ $\mathrm{Hg}$ at least twice before the stroke); (2) diabetes mellitus (known fasting hyperglycaemia before the stroke); (3) current or former cigarette smoking; (4) hypercholesterolaemia (cholesterol concentration $>6.5 \mathrm{mmol} / \mathrm{l}$ ).

For evaluating the functional disability after the stroke (activities of daily living, ADL), which was evaluated at the time of discharge, we used a five level classification; 1 for no disability, 2 for mild disability (return to all activities but with some difficulty), 3 for moderate disability (return to most main activities but with difficulty), 4 for severe disability (impossible to return to most activity), and 5 for death. ${ }^{15}$ The medication at the time of recurrence was also investigated.

Statistical comparisons among the groups were performed using the Kruskal-Wallis test for continuous variables, as well as the $\chi^{2}$ and Fisher's exact tests for categorical variables. Intergroup comparisons were performed with Bonferonni's method. A two tailed probability value $<0.05$ was considered significant.

\section{Results}

GENERAL FEATURES

During the study period, 102 patients (77 males and 25 females, mean age (SD): 63.7 (13.2) years) had 121 episodes of recurrent stroke (one recurrence in 86 patients, two in 14 patients, three in one patient, and four in one patient). Table 1 shows the stroke subtypes. There was no significant difference in age between men and women or among cardioembolic infarction, non-lacunar non-cardioembolic infarction, lacunar infarction, and brain haemorrhage. The mean interval between the first and last stroke tended to be longer in patients with initial lacunar infarction than in other groups.

\section{RISK FACTORS}

In the patients who had experienced a cardioembolic infarction as their first stroke, the frequency of cardiac ischaemia was significantly higher than in patients with an initial lacunar or haemorrhagic stroke $(\mathrm{p}<0.05)$. No patient with an initial brain haemorrhage had a potential cardiac source of embolism, which 
Table 2 Risk factors and the first stroke type

\begin{tabular}{|c|c|c|c|c|c|c|c|c|}
\hline & \multicolumn{2}{|c|}{$N L-C E$} & \multicolumn{2}{|c|}{$N L-N E$} & \multicolumn{2}{|l|}{$L I$} & \multicolumn{2}{|l|}{$B H$} \\
\hline & $n$ & $\%$ & $n$ & $\%$ & $n$ & $\%$ & $n$ & $\%$ \\
\hline Hypertension & 13 & 65.0 & 39 & 83.0 & 12 & 63.2 & 10 & 62.5 \\
\hline Diabetes mellitus & 5 & 25.0 & $7^{\star}$ & 14.9 & 8 & 42.1 & 3 & 18.6 \\
\hline Smoking & 7 & 35.0 & 29 & 61.7 & 7 & 36.8 & 6 & 37.5 \\
\hline Hypercholesterolaemia & 7 & 35.0 & 18 & 38.3 & 7 & 36.8 & 4 & 25.0 \\
\hline Cardiac ischaemia & 10 & 50.0 & 12 & 25.5 & $3 \rrbracket$ & 15.8 & $2 \S$ & 12.5 \\
\hline PCS & 20 & 100 & $16 \dagger \neq$ & 34.0 & $9 \dagger \neq$ & 47.4 & $0 \dagger$ & 0.0 \\
\hline \multirow{2}{*}{\multicolumn{9}{|c|}{$\begin{array}{l}{ }^{\star} \mathrm{p}<0.05 ; \text { lower than LI. } \\
\dagger \mathrm{p} \leqslant 0.0001 ; \text { lower than NL-CE. } \\
\ddagger \mathrm{p}<0.01 ; \text { higher than } \mathrm{BH} \text {. } \\
\mathrm{Sp}<0.05 ; \text { lower than NL-CE. } \\
\text { NL-CE=non-lacunar cardioembolic infarction; NL-NE=non-lacunar non-cardioembolic infarc- } \\
\text { tion; LI=lacunar infarction; BH=brain haemorrhage; PCS=potential cardiac source. } \\
\text { Table } 3 \text { Change in stroke type between first and recurrent stroke (number of episodes) }\end{array}$}} \\
\hline & & & & & & & & \\
\hline \multicolumn{2}{|l|}{ Recurrence $\backslash 1$ st } & \multicolumn{2}{|l|}{$N L-C E$} & \multicolumn{2}{|l|}{$N L-N E$} & $L I$ & \multicolumn{2}{|c|}{$B H$} \\
\hline \multicolumn{2}{|l|}{ NL-CE } & \multicolumn{2}{|l|}{$17^{\star}$} & \multicolumn{2}{|l|}{7} & 5 & \multicolumn{2}{|c|}{0} \\
\hline & \multicolumn{2}{|l|}{4} & \multicolumn{2}{|l|}{$33^{\star}$} & 9 & \multicolumn{2}{|c|}{3} \\
\hline & \multicolumn{2}{|l|}{1} & \multicolumn{2}{|l|}{7} & $14^{\star}$ & \multicolumn{2}{|c|}{5} \\
\hline \multicolumn{2}{|l|}{$\begin{array}{l}\mathrm{BH} \\
\text { Total }\end{array}$} & \multicolumn{2}{|l|}{0} & \multicolumn{2}{|l|}{4} & 1 & \multicolumn{2}{|c|}{$11^{\star}$} \\
\hline Total & & \multicolumn{2}{|l|}{22} & \multicolumn{2}{|l|}{51} & 29 & \multicolumn{2}{|c|}{19} \\
\hline
\end{tabular}

${ }^{\star} \mathrm{p}<0.001$; recurrence of the same type of stroke more often than other types.

NL-CE=non-lacunar cardioembolic infarction; NL-NE=non-lacunar non-cardioembolic infarction; $\mathrm{LI}=$ lacunar infarction; $\mathrm{BH}=$ brain hemorrhage; 1 st and recurrence=the first and recurrent stroke type; respectively. Bold numbers indicate recurrent episodes of the same type of stroke as the first.

was a significantly lower occurrence than in the other groups $(\mathrm{p}<0.01)$. Patients with an initial lacunar infarction were significantly more likely to have diabetes mellitus than those with nonlacunar non-cardioembolic infarction $(\mathrm{p}<0.05$, table 2). Some patients had multiple risk factors: $30 \%$ of the patients with initial cardioembolic infarction, $26 \%$ of those with initial non-lacunar non-cardioembolic infarction, $37 \%$ of those with initial lacunar infarction, and $6 \%$ of those with initial brain haemorrhage had three or more risk factors.

RECURRENT PATTERNS

In general, recurring strokes were always more often of the same type as the first stroke. The patients with an initial cardioembolic infarction had the highest same type recurrence rate, followed by those with initial non-lacunar noncardioembolic infarction, those with initial brain haemorrhage, and those with initial lacunar infarction (table 3 ). Seven $(44 \%)$ of 16 patients with initial non-lacunar noncardioembolic infarction and five $(56 \%)$ of nine patients with initial lacunar infarction who had a potential cardiac source of embolism developed cardioembolic infarcts. The recurring strokes of patients with an initial brain haemorrhage included non-lacunar noncardioembolic infarction in $16 \%$, lacunar infarction in $26 \%$, and cardioembolic infarction in none, whereas no patient with initial cardioembolic infarction, $8 \%$ with initial nonlacunar non-cardioembolic infarction, and $4 \%$ with initial lacunar infarction developed brain haemorrhage. Among the 14 patients who had three strokes, one repeated two cardioembolic infarcts, six repeated two non-lacunar noncardioembolic infarcts, five repeated two lacunar infarcts, one repeated two brain haemorrhages, and one experienced three different types of stroke (one non-lacunar non- cardioembolic infarct, one lacunar infarct, and one brain haemorrhage). The two patients who had more than three recurrences had experienced a lacunar infarction as their first stroke. One patient had four strokes which were all lacunar infarcts, and the other with five strokes had one non-lacunar non-cardioembolic infarct and four lacunar infarcts.

\section{CARDIOEMBOLIC INFARCTION}

In the 17 recurrent episodes of cardioembolic infarction, $10(76 \%)$ were located in the anterior circulation. Eight $(80 \%)$ of 10 patients with initial lesions in the anterior circulation and five $(71 \%)$ of seven patients with initial lesions in the posterior circulation developed recurrent episodes in the anterior circulation. No cardioembolic infarct was located in a border zone. Two of three patients who developed recurrent non-lacunar non-cardioembolic infarcts had a border zone infarction associated with carotid artery stenosis.

NON-LACUNAR NON-CARDIOEMBOLIC INFARCTION In all 84 patients with non-lacunar noncardioembolic infarcts, the most frequent cause was large artery atheroma, followed by atherosclerosis without more than $50 \%$ stenosis, and other causes $(39 \%, 37 \%$, and $24 \%$ respectively). Of the 12 patients with first stroke due to other causes, four patients had an embolic stroke from an undetermined source. Three of them developed embolic strokes from an undetermined source. The fourth patient developed a cardioembolic infarction. Two patients with internal carotid artery dissection did not have dissection as the cause of recurrent stroke: one patient had a non-lacunar non-cardioembolic infarct in the posterior circulation and the other a brain haemorrhage. Of the remaining six patients, one had positive anticardiolipin antibodies and one had hypertension as a sole risk factor.

Overall, infarcts most often involved the anterior circulation, followed by the posterior circulation and the border zone $(66 \%, 24 \%$, and $11 \%$ respectively). There was no significant relation between the involved territory and the presumed cause of the non-lacunar noncardioembolic infarction, except that border zone infarcts were typically associated with large artery stenosis or occlusion (91.7\%). Eighty nine per cent of initial episodes located in the anterior circulation, $63 \%$ of those located in the posterior circulation, and $50 \%$ of those located in the boder zone resulted in repeated recurrent episodes in the same territory $(p<0.01$ for the anterior circulation infarction, $p<0.05$ for the posterior circulation and the border zones). There were too few patients with subcortical versus superficial infarction to perform a meaningful analysis.

\section{LACUNAR INFARCTION}

The first stroke was a supratentorial lacunar infarct in 13 patients, and an infratentorial lacunar infarct in four patients. The other two patients showed multiple lacunar infarcts on CT. The recurrent episodes were essentially the same between the patients with diabetic 
mellitus as a sole risk factor and other patients: the eight patients with diabetes mellitus as a sole risk factor developed three cardioembolic infarcts, four non-lacunar non-cardioembolic infarcts, one brain haemorrhage, and repeated four lacunar infarcts, whereas the other 11 patients developed three cardioembolic infarcts, four non-lacunar non-cardioembolic infarcts, and repeated 10 lacunar infarcts.

BRAIN HAEMORRHAGE

In the patients with an initial brain haemorrhage, $11(69 \%)$ had a lobar and five $(31 \%)$ a non-lobar location. Of all episodes of haemorrhage, $21(77.8 \%)$ were lobar. No infratentorial haemorrhage occurred in either the initial or recurrent episodes. No patient with an initial brain haemorrhage was undergoing anticoagulation therapy when any brain haemorrhage occurred. Three patients with a lobar haemorrhage as the first stroke had a new haemorrhage in the same region without visible vascular malformation. Of all episodes of haemorrhage, $52 \%$ of the lobar haemorrhages and $67 \%$ of the non-lobar haemorrhages occurred in hypertensive patients. Ten patients with a hypertensive brain haemorrhage as the first stroke had 12 recurrent episodes, including five haemorrhages $(42 \%)$, three non-lacunar noncardioembolic infarcts (25\%), and four lacunar infarcts $(33 \%)$. Six patients with a nonhypertensive brain haemorrhage as the first stroke had seven recurrent episodes, including six haemorrhages $(86 \%)$ and one lacunar infarct $(14 \%)$.

\section{PREVENTIVE TREATMENT}

Among the 86 patients with a first ischaemic stroke, 58 patients $(67 \%)$ received antiplatelet drugs, 12 patients (14\%) received anticoagulant drugs (including those given for reasons other than stroke, such as the secondary prevention of pulmonary embolism), five patients $(6 \%)$ had surgical treatments, and 11 patients $(13 \%)$ had no special treatment for secondary prevention after the first episode. Only one patient developed a brain haemorrhage while on anticoagulation. Among the 16 patients who had at least two cardioembolic infarcts, 13 did not receive anticoagulant drugs at the time of recurrence. Of the two patients who had a carotid endarterectomy after a first non-lacunar non-cardioembolic infarction, one had a new stroke contralateral to the endarterectomy, and the other repeated a same type stroke on the ipsilateral side, associated with restenosis of the internal carotid artery.

Among the 16 patients with a first brain haemorrhage, two patients $(13 \%)$ received antiplatelet drugs, one patient $(6 \%)$ underwent a surgical treatment, and 13 patients $(81 \%)$ had no treatment after the first episode.

PROGNOSIS

After the first stroke, all but one of the patients $(95 \%)$ with an initial lacunar infarction were completely independent (level 1), whereas 65\% of the patients with an initial cardioembolic infarction, $49 \%$ of those with an initial non-lacunar non-cardioembolic infarction, and
$69 \%$ of those with brain haemorrhage were mildly to severely dependent (level 2-4). This difference in ADL between initial lacunar infarction and the other three subtypes was highly significant $(p<0.001)$. After a recurrent stroke, however, this difference disappeared $(p=0.26)$. The ADL was not significantly different between the patients with recurrence ipsilateral to the initial stroke and those with contralateral recurrence.

\section{Discussion}

Although there have been several prospective studies which have identified specific risk factors, aetiological features, and prognostic characteristics for recurrent strokes, the results are heterogeneous and remain controversial. ${ }^{7916}$ Identification of the underlying mechanisms for recurrence is the key to effective secondary prevention. However, a precise aetiological classification of recurrent episodes in a large number of patients has not been available from hospital or community based stroke registries, although there are scarce smaller studies, which primarily focused on lacunar stroke. ${ }^{17-23}$ The present study is not an epidemiological study evaluating the frequency and risk of stroke recurrence, but an attempt at better analysing the different pathophysiological patterns of first versus second and further strokes.

Our findings show that despite preventive treatment, patients with first stroke may not only experience a repeat of the same type of stroke, but may also develop other kinds of strokes. Previous hospital or community based follow up studies did not emphasise the potential changes in recurrent stroke subtypes, and our study provides a first attempt at analysing the patterns of recurrent stroke and delineating the factors associated with recurrence in each subtype.

The present study has inevitable limitations. Firstly, because this is not a prospective study, it is difficult to determine the true recurrence rate in patients with each subtype of stroke. Patients might neglect very mild symptoms, which may decrease the apparent rate of recurrence of lacunar stroke. Fatal recurrence episodes might not have been referred to our service, which might be a cause of the apparent lack of pontine haemorrhage. Secondly, the whole group of selected patients might have some bias because of the strict exclusion criteria. A mean age of 64 years seems slightly young compared with other community based and hospital based studies. However, the mean age of the first 1000 consecutive patients registered in the Lausanne Stroke Registry was 61.1 (SD 12.2) years, ${ }^{13}$ which suggests that the patients selected for the present study may not significantly vary from the whole group of the Lausanne Stroke Registry, at least physically. Another limitation is the classification of the recurrence episodes. It is always difficult to know the true aetiology of a stroke in patients if there are two or more potential causes. To make the mechanism of stroke recurrence as clear as possible, we tried to classify all the episodes into either of the subgroups. It may produce some bias. For example, as cardiac 
embolic sources may not disappear, it may mask the true cause of recurrent episodes. It is especially difficult to discriminate non-lacunar non-cardioembolic infarction from nonlacunar cardioembolic infarction and it is possible that some recurrences of non-lacunar non-cardioembolic infarction were classified as non-lacunar cardioembolic infarcts. The opposite is less likely because our criteria tend to classify more infarcts which occurred to cases with cardioembolic sources into cardioembolic infarction. It is also difficult to detect the lesions responsible for recurrence when former ischaemic lesions are visible on the neuroradiological images. We tried to detect these earlier lesions based on clinical symptoms. However, a lacunar recurrence occurring in the same side as the first lacunar episode may not be taken as a recurrent episode, but a worsening of the sequelae. It may make the same type recurrence rate of lacunar infarction lower than the other type recurrence rate of this group. Despite these limitations, however, we think that the present study can provide valuable information about recurrent mechanisms, as there have been no other studies examining the aetiology of the first and all recurrent episodes in detail.

Indeed, our study emphasises particular risk factors associated with recurrence, such as cardiac ischaemia with cardioembolic infarction, diabetes mellitus with lacunar infarction, and the absence of a potential cardiac source of embolism with brain haemorrhage. It also underlines, as Sobel et al have reported, ${ }^{24}$ the importance of the combination of risk factors for stroke recurrence. The patients with lacunar infarction had the highest number of risk factors, whereas the patients with brain haemorrhage had the lowest number.

There are reports suggesting that a recurrent stroke is usually of the same type as that of the first stroke. ${ }^{3}{ }^{7}$ In the present study, this was primarily true for non-lacunar non-cardioembolic infarction in general and for cardioembolic infarction in particular, but very often $(38 \%)$ the recurrent stroke was not of the same type as the first stroke, particularly for lacunar infarction (52\%).

Some studies have reported a predominance of non-lacunar non-cardioembolic stroke recurrence in the same vascular territory. ${ }^{125}$ These studies, however, considered only the anterior versus posterior circulation. We found a significantly higher same territory recurrence rate within the anterior circulation, posterior circulation, and border zone compared with the recurrence rate in territories other than the one involved in the first stroke.

Half of the patients with lacunar infarction developed non-lacunar infarction, including cardioembolic stroke. Prospective studies have reported that $32 \%-40 \%$ of the patients with lacunar infarction may develop non-lacunar non-cardioembolic infarction, which is higher than the rate of subsequent lacunar infarction in patients with a non-lacunar non-cardioembolic infarction as the first stroke, ${ }^{17}{ }^{18} 26$ as we found in the present study. Kappelle $e t a l^{26}$ have hypothesised a common aetiology between large artery and small artery disease, with coexisting large artery disease in patients with symptomatic small artery disease, or the association of a potential cardiac source with small artery disease in some patients, or a more positive preventive effect of aspirin for stroke due to small artery disease. Another possibility is that either large vessel disease or cardiac embolism may already play an aetiological role in some initial strokes labelled as lacunar infarction, ${ }^{27}$ and subsequently also in recurrent strokes. Our findings show that in secondary prevention after lacunar infarction, large artery disease should also be considered, especially in patients with multiple risk factors who may have a pathological basis not only for small artery disease, but also for large artery disease.

Recently, several authors have noted that the functional recovery of patients with lacunar infarction may be better than in patients with a non-lacunar noncardioembolic infarction. ${ }^{17} 182829$ In our study, the excellent ADL of the lacunar infarction group after the first stroke decreased markedly after recurrence. The importance of secondary prevention of lacunar infarction should be emphasised based on the resulting quality of life.

As already shown in recent reports, recurrent haemorrhage is not uncommon. ${ }^{30}{ }^{31}$ We found a high proportion of lobar locations for first and recurrent haemorrhages but no infratentorial haemorrhage, the second probably because of lower incidence and higher mortality rates. ${ }^{32} 33$ Our findings emphasise the potentially different aetiologies between first ever and recurrent brain haemorrhage. Hypertension may be the most predominant aetiology of first ever brain haemorrhage. First ever brain haemorrhage occurs predominantly in deep in the cerebral hemispheres, with rupture of small, perforating arteries which are the target of chronic hypertension. ${ }^{34}$ The reported rate of lobar location for primary brain haemorrhage is $20 \%$ $55 \%$, which is linked to the underlying aetiology. ${ }^{13} 323^{33}{ }^{35-37}$ In an earlier reported study from our institution, the proportion of lobar locations for first haemorrhage was $40 \%{ }^{13}$ including cases with angioma, which constitute a subgroup with a particularly high potential for local rebleeding. In a study of recurrent brain haemorrhage due to hypertension, ${ }^{31}$ badly controlled in most of the patients, only $8.5 \%$ of recurrences were lobar. Our high proportion of lobar location may indicate aetiologies other than hypertension, such as small angioma or cerebral amyloid angiopathy. Passero et $a l^{30}$ have found that a lobar location for a first haemorrhage is a predictor of rebleeding, although they did not specify if the recurrent haemorrhage was lobar or whether or not an underlying angioma was found. We also found a predominance of recurrent haemorrhage in patients with a non-hypertensive haemorrhage as the first stroke. Cerebral amyloid angiopathy ${ }^{38}$ and

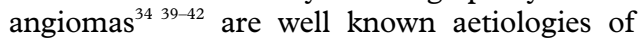
non-hypertensive haemorrhage, usually in a lobar location. Three of our patients had recurrent lobar haemorrhage at the same site, which might be explained by a small, hardly visible angioma. ${ }^{41}$ Because lobar haemorrhage 
recurred in different sites in many patients, CAA may also play a part, although no histological proof was available.

Our findings show that recurrence of stroke need not be of the same type, aetiology, and location as the first episode. Many patients in the present study had multifactorial risk factors and coexisting potential causes of stroke, explaining in part why the patterns of stroke recurrence may be so heterogeneous. An improved understanding of the patterns of recurrence of stroke may allow for the development of more accurate prediction and better prevention of recurrence in individual patients.

We thank Drs S Blecic and JP Neau for their help in selecting the patients.

1 Bronner LL, Kanter DS, Manson JE. Primary prevention of stroke. N Engl f Med 1995;333:1392-400.

2 Meissner I, Whisnant JP, Garraway WM. Hypertension management and stroke recurrence in a community management and stroke recurrence in a community
(Rochester, Minnesota, 1950-79). Stroke 1988;19:459-63.

3 Sacco RL, Wolf PA, Kannel WB, et al. Survival and Sacco RL, Wolf PA, Kannel WB, et al. Survival and
recurrence following stroke-the Framingham study. recurrence following
Stroke 1982;13:290-5.

4 Alter M, Sobel E, McCoy RL, et al. Stroke in the Lehigh Valley: risk factors for recurrent stroke. Neurology 1987;37: 503-7.

5 Viitanen M, Eriksson S, Asplund K. Risk of recurrent stroke, myocardial infarction and epilepsy during long term follow up after stroke. Eur Neurol 1988;28:227-31.

6 Sacco RL, Foulkes MA, Mohr JP, et al. Determinants of early recurrence of cerebral infarction-the stroke data bank. Stroke 1989;20:983-9.

7 Hier DB, Foulkes MA, Swiontoniowski M, et al. Stroke recurrence within 2 years after ischemic infarction. Stroke 1991;22:155-61.

8 Broderick JP, Phillips SJ, O'Fallon WM, et al. Relationship of cardiac disease to stroke occurrence, recurrence, and of cardiac disease to stroke occu
mortality. Stroke 1992;23:1250-6.

9 Burn J, Dennis M, Bamford J, et al. Long term risk of recurrent stroke after a first ever stroke-the Oxfordshire rent stroke after a first ever stroke-the Ox
community stroke project. Stroke 1994;25:333-7.

10 Lai SM, Alter M, Friday G, et al. A multifactorial analysis of risk factors for recurrence of ischemic stroke. Stroke 1994; 25:958-62

11 Alter M, Friday G, Min Lai S, et al. Hypertension and risk of stroke recurrence. Stroke 1994;25:1605-10.

2 Irie K, Yamaguchi T, Minematsu K, et al. The J-curve phenomenon in stroke recurrence. Stroke 1993;24:1844-9.

13 Bogousslavsky J, Van Mell G, Regli F. The Lausanne Stroke Registry: analysis of 1000 consecutive patients with first stroke. Stroke 1988;19:1083-92.

14 Bogousslavsky J, Regli F. Borderzone infarction distal to internal carotid artery occlusion: prognostic implications. Ann Neurol 1986;20:346-50.

15 NINCDS Ad Hoc Committee on Cerebrovascular Diseases. A classification and outline of cerebrovascular eases. A classification and outline
diseases 2. Stroke 1975;6:565-616.

16 Sacco RL. Risk factors and outcomes for ischemic stroke.

Neurology 1995;45(suppl 1):S10-4.
17 Nadeau SE, Jordan JE, Mishra SK, et al. Stroke rates in patients with lacunar and large vessel cerebral infarctions. $\mathcal{f}$ Neurol Sci 1993;114:128-37.

18 Clavier I, Hommel M, Besson G, et al. Long term prognosis of symptomatic lacunar infarcts - a hospital-based study. Stroke 1994;25:2005-9.

19 Boiten J, Lodder J. Prognosis for survival, handicap and recurrence of stroke in lacunar and superficial infarction. Cerebrovasc Dis 1993;3:221-6.
20 Gandolfo C, Moretti C, Dall'Agata D, et al. Long. term prognosis of patients with lacunar syndromes. Acta Neural Scand 1986;74:224-9.

21 Miyao S, Takano A, Teramoto J, et al. Leukoaraiosis in relation to prognosis for patients with lacunar infarction. Stroke 1992;23:1434-8.

22 Salgado AV, Ferro JM, Couveia-Oliveira A. Long term prognosis of first ever lacunar stroke. A hospital based study. Stroke 1996;27:661-6.

23 Samuelsson M, Lindell D, Norrving B. Presumed pathogenetic mechanisms of recurrent stroke after lacunar infarction. Cerebrovasc Dis 1996;6:128-36.

24 Sobel E, Alter M, Davanipour Z, et al. Stroke in the Lehigh Valley: combined risk factors for recurrent ischemic stroke. Neurology 1989;39:669-72.

25 Cillessen JPM, Kappelle LJ, van Swieten JC, et al. Does cerebral infarction after a previous warning occur in the sam vascular territory? Stroke 1993;24:351-54.

26 Kappelle LJ, van Latum JC, Algra A, et al, Dutch TIA Trial Study Group. Recurrent stroke after transient ischemic attack or minor ischemic stroke: does the distinction between small and large vessel disease remain true to type? f Neurol Neurosurg Psychiatry 1995;59:127-31.

27 Bogousslavsky J, Cachi C, Regli F, et al. Cardiac sources of embolism and cerebral infarction- clinical consequences and vascular concomitants: the Lausanne stroke registry. Neurology 1991;41:855-9.

28 Landi G, Cella E, Boccardi E, et al. Lacunar versus non-lacunar infarcts: pathogenetic and prognostic differences. F Neurol Neurosurg Psychiatry 1992;55:441-5.

29 Sacco RL, Shi T, Zamanillo MC, et al. Predictors of mortality and recurrence after hospitalized cerebral infarction in an urban community: the Northern Manhattan stroke study. Neurology 1994;44:626-34.

30 Passero S, Burgalassi L, D'Andrea P, et al. Recurrence of bleeding in patients with primary intracerebral hemorrhage. Stroke 1995;26:1189-92.

31 Chen ST, Chiang CY, Hsu CY, et al. Recurrent hypertensive intracerebral hemorrhage. Acta Neurol Scand 1995;91:128-

32 Fogelholm R, Nuutila M, Vuorela A-L. Primary intracerebral haemorrhage in the Jyväskylä region, Central Finland, 1985-89: incidence, case fatality rate, and functional

33 Boonyakarnkul S, Dennis M, Sandercock P, et al. Primary ntracerebral haemorrhage in the Oxfordshire community stroke project 1 . incidence, clinical features and causes. Cerebrovasc Dis 1993;3:343-9.

34 Kase CS, Mohr JP, Caplan LR. Intracerebral hemorrhage. In: Barnett HJM, Mohr JP, Stein BM, et al, eds. Stroke, 2nd ed. New York: Churchill Livingstone, 1992:561-616.

35 Fieschi C, Carolei A, Fiorelli M, et al. Changing prognosis of primary intracerebral hemorrhage: results of a clinical and computed tomographic follow up study of 104 patients. Stroke 1988;19:192-5.

36 Franke CL, van Swieten JC, Algra A, et al. Prognostic factors in patients with intracerebral haematoma. F Neurol Neurosurg Psychiatry 1992;55:653-657.

37 Helweg-Larsen S, Sommer W, Strange P, Lester J, Boysen G. Prognosis for patients treated conservatively for spontaneous intracerebral hematomas. Stroke 1984;15:1045-8.

38 Vinters HV. Cerebral amyloid angiopathy. In: Barnett HJM, Mohr JP, Stein BM, et al, eds. Stroke 2nd ed. New York: Charchill Livingstone, 1992: 821-58.

39 Margolis G, Odom GL, Woodhall B, et al. The role of small angiomatous malformations in the production of intracerebral hematomas. I Neurosurg 1951;8:564-75.

40 Crawford JV, Russell DS. Cryptic arteriovenous hamartomas of the brain. $\mathcal{F}$ Neurol Neurosurg Psychiatry 1956;19:111

41 Becker DH, Townsend JJ, Kramer RA, et al. Occult cerebrovascular malformations-a series of 18 histologically verified cases with negative angiography. Brain 1979; 102:249-87.

42 Tanaka Y, Furuse M, Iwasa H, et al. Lobar intracerebral hemorrhage: etiology and a long term follow up study of 32 patients. Stroke 1986;17:51-7. 